\title{
A Comparison Between Star Products on Regular Orbits of Compact Lie Groups
}

\author{
R. Fioresi*円 and M. A. Lledó ${ }^{\dagger}$ \\ * Dipartimento di Matematica, Università di Bologna \\ Piazza di Porta S. Donato, 5. \\ 40126 Bologna. Italy. \\ e-mail: fioresi@dm.UniBo.it \\ $\dagger$ Dipartimento di Fisica, Politecnico di Torino, \\ Corso Duca degli Abruzzi 24, I-10129 Torino, Italy, and \\ INFN, Sezione di Torino, Italy. \\ e-mail: lledo@athena.polito.it
}

\begin{abstract}
In this paper an algebraic star product and differential one defined on a regular coadjoint orbit of a compact semisimple group are compared. It is proven that there is an injective algebra homomorphism between the algebra of polynomials with the algebraic star product and the algebra of differential functions with the differential star product structure.
\end{abstract}

\footnotetext{
${ }^{1}$ Investigation supported by the University of Bologna, funds for selected research topics.
} 


\section{Introduction}

The problem of classification of differential star products on a general Poisson manifold was solved in Ref. [1]. The existence of star products on symplectic manifolds was already proven in Ref. [2, 3] and, using a different technique, a construction of a star product and a classification of all star products on a symplectic manifold was given in Ref. [4. 5]. For other special cases, as for regular manifolds, a proof of existence of tangential star products was known (see Ref. 6]).

To motivate our discussion, let us consider the Heisenberg group $H=\mathbb{R}^{3}$ with multiplication

$$
\left(a_{1}, b_{1}, c_{1}\right) \cdot\left(a_{2}, b_{2}, c_{2}\right)=\left(a_{1}+a_{2}, b_{1}+b_{2}, c_{1}+c_{2}+a_{1} b_{2}\right) .
$$

Its Lie algebra is $\mathfrak{h}=\mathbb{R}^{3}=\operatorname{span}\left\{Q, P, E^{\prime}=-i E\right\}$ with commutation rules

$$
[Q, P]=-i E \quad(\text { the rest trivial) }
$$

The coadjoint orbits of $H$ are the planes $c=$ constant $\neq 0$ (regular orbits) and the points $(a, b, 0)$. One way of obtaining the Moyal-Weyl product on $\mathbb{R}^{2}$ is considering the Weyl map or symmetrizer in the enveloping algebra of $\mathfrak{h}$,

$$
\begin{aligned}
\text { Sym }: \operatorname{Pol}\left[\mathfrak{h}^{*}\right] & \rightarrow U(\mathfrak{h}) \\
x_{1} x_{2} \cdots x_{k} & \mapsto \frac{1}{k !} \sum_{\sigma \in S_{k}} X_{\sigma(1)} \cdots X_{\sigma(k)},
\end{aligned}
$$

where $x_{i}$ are the coordinates on $\mathfrak{h}^{*}$ on the basis dual to $\left\{X_{i}\right\}$ in $\mathfrak{h}$. By multiplying the commutation rules by a formal parameter $h$ we obtain the following star product on $\mathrm{Pol}\left[\mathfrak{h}^{*}\right][[h]]$

$$
f \star g=\operatorname{Sym}^{-1}(\operatorname{Sym}(f) \operatorname{Sym}(g)) .
$$

This star product is differential, so it can be extended to $C^{\infty}\left(\mathfrak{h}^{*}\right)$, it is tangential, so it can be restricted to the orbits and it is algebraic, that is, it is closed (and convergent) on polynomials.

If instead of the Heisenberg group we take another group, say $\mathrm{SU}(2)$, we can define a star product using the symmetrizer as is (1). The resulting star product is algebraic and differential but it is not tangent to the coadjoint orbits, so it does not define a star product on them, in this case the spheres. 
Two different approaches can be taken at this point. One is to look for a differential star product on the sphere in the spirit of Refs. [2, 3, 4, 1]. The resulting star product is neither algebraic nor appears related to the product on the enveloping algebra. The other approach insists on using the product in the enveloping algebra. The consequence is that differentiability is lost. This kind of star products have been considered in Refs. [7, 8, 9, 10] and in particular, in Refs. 8, 9] it was proven that a non differential star product on coadjoint orbits of $\mathrm{SU}(2)$ corresponds to the standard quantization of angular momentum. It seems then unavoidable to look to a wider class of star products than the differential ones. In particular, one cannot immediately assume that the canonical quantization given by Kontsevich's theorem [1] is the one relevant for physics in all cases.

The problem of existence and classification of algebraic star products on algebraic Poisson varieties appears as a separate problem, mathematically interesting in itself, which has been recently studied in Ref.[11. From the physical point of view it is of interest since the algebra of a physical quantum system may have a non differential star product, as in the case of the angular momentum and its standard quantization.

Our purpose here is to compare the deformations obtained by algebraic 8, 9] and by differential methods on regular coadjoint orbits of compact semisimple Lie groups. We want to establish if there is some kind of equivalence among these different star products. We work with a family of algebraic star products, not all isomorphic, and we relate them to the differential star product given by Kontsevich's theorem or Fedosovs's construction [4]. Our result is that one of the algebraic star products can be injected homomorphically into the differential one.

The organization of the paper is as follows. In section 2 we recall known facts concerning coadjoint orbits of a semisimple compact group $G$ and its complexification $G_{\mathbb{C}}$. In section 3 we introduce different star products on a fixed regular coadjoint orbit $\Theta$ and on a tubular neighborhood of the orbit $\mathcal{N}_{\Theta}$. and we prove that two different star products on $\mathcal{N}_{\Theta}$, one tangential $\star_{T}$ and one not tangential $\star_{S \mathcal{N}_{\Theta}}$, are equivalent. In section 4 we show our main result, that there is an injective homomorphism between an algebraic star product $\star_{P \Theta}$ and a differential one $\star_{T \Theta}$ on the orbit $\Theta$. The algebraic star product belongs to the family constructed in [8], while the differential one is obtained by gluing tangential star products defined on open sets of $\mathcal{N}_{\Theta}$, computed with Kontsevich's formula [1]. In Appendix A we give for completeness some standard definitions and results on star products and 
deformations. In Appendix B we give an explicit formula for the gluing of star products given in open sets and satisfying a compatibility condition, in terms of a partition of unity.

\section{Coadjoint orbits of semisimple Lie groups}

Let $G$ be a compact semisimple group of dimension $n$ and rank $m$ and $\mathfrak{g}$ its Lie algebra. Let $\mathfrak{g}^{*}$ be the dual of $\mathfrak{g}$. On $C^{\infty}\left(\mathfrak{g}^{*}\right)$ we have the Kirillov Poisson structure:

$$
\left\{f_{1}, f_{2}\right\}(\lambda)=\left\langle\left[\left(d f_{1}\right)_{\lambda},\left(d f_{2}\right)_{\lambda}\right], \lambda\right\rangle, \quad f_{1}, f_{2} \in C^{\infty}\left(\mathfrak{g}^{*}\right), \quad \lambda \in \mathfrak{g}^{*} .
$$

$(d f)_{\lambda}: \mathfrak{g}^{*} \rightarrow \mathbb{R}$ can be considered as an element of $\mathfrak{g}$, and $[$,$] is the Lie bracket$ on $\mathfrak{g}$. Let $\left\{X_{1} \ldots X_{n}\right\}$ be is a basis of $\mathfrak{g}$ and $\left\{x^{1}, \ldots x^{m}\right\}$ the coordinates on $\mathfrak{g}^{*}$ in the dual basis. We have that

$$
\left\{f_{1}, f_{2}\right\}\left(x^{1}, \ldots x^{n}\right)=\sum_{i j k} c_{i j}^{k} x^{k} \frac{\partial f_{1}}{\partial x^{i}} \frac{\partial f_{2}}{\partial x^{j}},
$$

where $c_{i j}^{k}$ are the structure constants of $\mathfrak{g}$, that is $\left[X_{i}, X_{j}\right]=\sum_{k} c_{i j}^{k} X_{k}$.

$\mathfrak{g}^{*}$ is an algebraic Poisson manifold since the ring of polynomials $\mathbb{R}\left[\mathfrak{g}^{*}\right]$, is closed under the Poisson bracket.

The Kirillov Poisson structure is neither symplectic nor regular. The symplectic leaves are the orbits of the coadjoint action of $G$ on $\mathfrak{g}^{*}$,

$$
\left\langle\operatorname{Ad}^{*}(g) \lambda, Y\right\rangle=\left\langle\lambda, \operatorname{Ad}\left(g^{-1}\right) Y\right\rangle \quad \forall g \in G, \quad \lambda \in \mathfrak{g}^{*}, \quad Y \in \mathfrak{g} .
$$

We denote by $\Theta_{\lambda}$ the orbit of an element $\lambda \in \mathfrak{g}^{*}$ under the coadjoint action.

Let $G_{\mathbb{C}}$ be the complexification of $G$ and let $\mathfrak{g}_{\mathbb{C}}$ be its Lie algebra. Let $\Theta_{\lambda \mathbb{C}}$ be the coadjoint orbit of $\lambda \in \mathfrak{g}^{*}$ in $\mathfrak{g}_{\mathbb{C}}^{*}$ under the action of $G_{\mathbb{C}}$. $\Theta_{\lambda \mathbb{C}}$ is an algebraic variety defined over $\mathbb{R}$ and $\Theta_{\lambda}=\Theta_{\lambda \mathbb{C}} \cap \mathfrak{g}^{*}$.

Let $\mathbb{C}\left[\mathfrak{g}^{*}\right]$ be the ring of polynomials on $\mathfrak{g}_{\mathbb{C}}^{*}$. We denote by $\operatorname{Inv}\left(\mathfrak{g}_{\mathbb{C}}^{*}\right)$ the subalgebra of polynomials invariant under the coadjoint action. It is generated by homogeneous polynomials, $p_{i}, i=1, \ldots m$, (Chevalley generators). We have that

$$
\operatorname{Inv}\left(\mathfrak{g}^{*}\right)=\operatorname{Inv}\left(\mathfrak{g}_{\mathbb{C}}^{*}\right) \cap \mathbb{R}\left[\mathfrak{g}^{*}\right]
$$

If $\lambda$ is regular, the ideal of $\Theta_{\lambda \mathbb{C}}$ is given by $[12], \mathcal{I}_{0 \mathbb{C}}=\left(p_{i}-c_{i}, i=\right.$ $1 \ldots m), c_{i} \in \mathbb{R}$, and the polynomials on $\Theta_{\lambda \mathbb{C}}$ by $\mathbb{C}\left[\Theta_{\lambda \mathbb{C}}\right]=\mathbb{C}\left[\mathfrak{g}^{*}\right] / \mathcal{I}_{0 \mathbb{C}}$. For 
the real forms the ideal of $\Theta_{\lambda}$ is $\mathcal{I}_{0}=\mathcal{I}_{0 \mathbb{C}} \cap \mathbb{R}\left[\mathfrak{g}^{*}\right]$, with the same generators than the complex one and $\mathbb{R}\left[\Theta_{\lambda}\right]=\mathbb{R}\left[\mathfrak{g}^{*}\right] / \mathcal{I}_{0}=\mathbb{C}\left[\Theta_{\lambda \mathbb{C}}\right] \cap \mathbb{R}\left[\mathfrak{g}^{*}\right]$.

\section{Star products on a regular coadjoint orbit.}

In this section we will consider complex star products which are deformations of the complexification of a real Poisson algebra. We want to describe different star products [9] that will be later compared.

From now on we fix a regular coadjoint orbit $\Theta$ in $\mathfrak{g}^{*}$. We will consider $\mathfrak{g}_{h}$ the Lie algebra over $\mathbb{C}[[h]]$ obtained by multiplying the structure constants of $\mathfrak{g}_{\mathbb{C}}$ by a formal parameter $h . U_{h}$ is its enveloping algebra.

\section{The star products $\star_{S}$ and $\star_{S \mathcal{N}_{\Theta}}$}

It is well known that $U_{h}$ is a formal deformation of $\mathbb{C}\left[\mathfrak{g}^{*}\right]$. In Ref. [1] it was shown that this deformation is isomorphic to the star product canonically associated to the Kirillov Poisson structure. Moreover, since the linear coordinates on $\mathfrak{g}^{*}$ are global, one can compute a star product using Kontsevich's universal formula.

The symmetrizer Sym (1) (that can be defined in the same way for any Lie algebra) defines through (2) a differential and algebraic star product on $\mathfrak{g}^{*}$ that we denote by $\star_{S}$. Any other isomorphism that is the identity modulo $h$ could be chosen in the place of Sym. All the star products constructed in this way are equivalent to the one obtained with Kontsevich's explicit formula. All of them are algebraic and differential, but none of them is tangential to all the orbits [13].

Since a differential star product tangential to all orbits cannot exist in the whole $\mathfrak{g}^{*}$ (see appendix B), we have to look for a smaller space. We consider a regular orbit $\Theta$ and a regularly foliated neighborhood of the orbit, a tubular neighborhood $\mathcal{N}_{\Theta} \simeq \Theta \times \mathbb{R}^{m}$, where the global coordinates in $\mathbb{R}^{m}$ are the invariant polynomials $p_{i}, i=1, \ldots m$. Since $\star_{S}$ is differential, it can be restricted to the open set $\mathcal{N}_{\Theta}$. We will denote that restriction by $\star_{S \mathcal{N}_{\Theta}}$. $\star_{S \mathcal{N}_{\Theta}}$ is a differential star product belonging to the canonical equivalence class associated to the Kirillov Poisson structure restricted to $\mathcal{N}_{\Theta}$.

Since $\mathcal{N}_{\Theta}$ is a regular Poisson manifold, we know that a tangential star product (with respect to the symplectic leaves) exists [6]. We want to prove 
that there exists a tangential star product on $\mathcal{N}_{\Theta}$ equivalent to $\star_{S \mathcal{N}_{\Theta}}$.

\section{The star products $\star_{T}$ and $\star_{T \Theta}$}

We want to define a tangential star product $\star_{T}$ on $\mathcal{N}_{\Theta}$ and its restriction $\star_{T \Theta}$ to the regular orbit $\Theta$. We will use the gluing of star products computed in the Appendix B in terms of a partition of unity.

Let $\mathcal{U}=\left\{U_{r}, r \in J\right\}, J$ a set of indices, be a good covering of $\mathcal{N}_{\Theta}$ with Darboux charts. The coordinates in an open set $U_{r}$ are

$$
\begin{aligned}
& \varphi_{r}: U_{r} \longrightarrow \mathbb{R}^{n} \quad \text { with } \\
& \varphi_{r}=\left(\theta_{r}, \pi_{r}, p\right)=\left(\theta_{r}^{1}, \ldots, \theta_{r}^{(n-m) / 2}, \pi_{r}^{1}, \ldots, \pi_{r}^{(n-m) / 2}, p_{1}, \ldots, p_{m}\right), \\
& \left\{\theta_{r}^{\alpha}, \pi_{r}^{\beta}\right\}=\delta^{\alpha \beta}, \quad\left\{\theta_{r}^{\alpha}, p_{i}\right\}=0, \quad\left\{p_{i}, \pi_{r}^{\beta}\right\}=0 .
\end{aligned}
$$

The invariant polynomials $p_{i}$ are global coordinates, so $U_{r} \simeq \hat{U}_{r} \times \mathbb{R}^{m}$ and $\left\{\left(\hat{U}_{r},\left(\theta_{r}, \pi_{r}\right)\right)\right\}_{r \in J}$ is an atlas of $\Theta$, with $\left\{\hat{U}_{r},\left(\theta_{r}, \pi_{r}\right), r \in J\right\}$ the symplectic charts.

We can now apply Kontsevich's formula in a coordinate patch $U_{r}$, using the Darboux coordinates $\varphi_{r}$. We denote this star product by $\star_{r}^{K}$. It is a tangential star product. If $\star_{r}$ denotes the restriction of $\star_{S \mathcal{N}_{\Theta}}$ to $U_{r}$, then $\star_{r}$ and $\star_{r}^{K}$ are equivalent. We will denote by

$$
R_{r}:\left(C^{\infty}\left(U_{r}\right)[[h]], \star_{r}\right) \longrightarrow\left(C^{\infty}\left(U_{r}\right)[[h]], \star_{r}^{K}\right)
$$

the isomorphism

$$
R_{r}\left(f \star_{r} g\right)=R_{r}(f) \star_{r}^{K} R_{r}(g), \quad R_{r}=\mathrm{Id}+\sum_{i=1}^{\infty} h^{n} R_{r}^{n} .
$$

In the intersection $U_{r s}=U_{r} \cap U_{s}$ one has that $\star_{r}^{K}$ and $\star_{s}^{K}$ are equivalent as in (13) of Appendix B with

$$
T_{r s}=R_{r} \circ R_{s}^{-1} .
$$

We have the following

Proposition 3.1 Let $\mathcal{N}_{\Theta}$ and $\mathcal{U}$ be the tubular neighborhood of the orbit $\Theta$ and the covering of $\mathcal{N}_{\Theta}$ defined above. Let $\mathcal{F}_{S}$ be the sheaf of star products defined by $\star_{S N_{\Theta}}$ and $\star_{r}^{K}$ the star product obtained via Kontsevich formula in $U_{r} \in \mathcal{U}$. 
The assignment

$$
U_{r} \mapsto\left(C^{\infty}\left(U_{r}\right), \star_{r}^{K}\right) \quad \forall U_{r} \in \mathcal{U}
$$

is a sheaf of star products isomorphic to $\mathcal{F}_{S}$. There is a star product $\star_{T}$ on $\mathcal{N}_{\Theta}$ that is tangential and gauge equivalent to $\star_{S \mathcal{N}_{\Theta}}$.

Proof. It is immediate that the transition functions (3) satisfy the conditions (14) of Appendix B, so we have a sheaf of star products that we will denote by $\mathcal{F}_{T}$. The isomorphisms $R_{r}$ give the isomorphism of sheaves among $\mathcal{F}_{S}$ and $\mathcal{F}_{T}$.

Given a partition of unity subordinated to $\mathcal{U}$ one can use the method of Appendix B to construct a global star product. From the explicit formula (15), one can see that it is a tangential star product.

The restriction of $\star_{T}$ to the orbit will be denoted by $\star_{T \Theta}$.

\section{The star products $\star_{P}$ and $\star_{P \Theta}$}

We want to define an algebraic star product $\star_{P}$ on $\mathfrak{g}^{*}$ and its restriction to the orbit $\Theta$, the algebraic star product $\star_{P \Theta}$.

We consider the ideal in $U_{h}$

$$
\mathcal{I}_{h}=\left(P_{i}-c_{i}(h), i=1, \ldots m\right),
$$

where $P_{i}=\operatorname{Sym}\left(p_{i}\right)$ and $c_{i}(h) \in \mathbb{C}[[h]]$ with $c_{i}(0)=c_{i}^{0}$. It was proven in Ref. [8] that $U_{h} / \mathcal{I}_{h}$ is a deformation quantization of $\mathbb{C}[\Theta]=\mathbb{C}\left[\mathfrak{g}^{*}\right] / \mathcal{I}_{0}$ where

$$
\mathcal{I}_{0}=\left(p_{i}-c_{i}^{0}, i=1, \ldots m\right)
$$

is the ideal of a regular orbit $\Theta$. Further properties of this deformation where studied in Ref. [9]. The generalization of this construction to non regular orbits was done in Ref. 10.

A star product associated to this deformation can be constructed by giving a $\mathbb{C}[[h]]$-module isomorphism:

$$
\psi: \mathbb{C}\left[\mathfrak{g}^{*}\right][[h]] \longrightarrow U_{h}
$$

that maps the ideal $\mathcal{I}_{0}$ isomorphically onto $\mathcal{I}_{h}$. One way of choosing this map (but not the only one) is by using the decomposition of $\mathbb{C}\left[\mathfrak{g}^{*}\right]$ in terms of invariant and harmonic polynomials [12]

$$
\mathbb{C}\left[\mathfrak{g}^{*}\right] \cong \operatorname{Inv}\left(\mathfrak{g}_{\mathbb{C}}^{*}\right) \otimes \mathcal{H}
$$


The harmonic polynomials $\mathcal{H}$ are in one to one correspondence with $\mathbb{C}[\Theta]$ and we have a monomial basis $\mathcal{B}=\left\{x_{i_{1}} \ldots x_{i_{k}},\left(i_{1}, \ldots i_{k}\right) \in I\right\}$, where $I$ is some subset of indices such that $\mathcal{B}$ is a basis of $\mathbb{C}[\Theta]$ (see Ref. [8] for more details). We consider the following $\mathbb{C}[[h]]$-module isomorphism:

$$
\begin{aligned}
\psi: \mathbb{C}\left[\mathfrak{g}^{*}\right][[h]] & \longrightarrow U_{h} \\
\left(p_{i_{1}}-c_{i_{1}}^{0}\right) \cdots\left(p_{i_{k}}-c_{i_{k}}^{0}\right) \otimes x_{j_{1}} \cdots x_{j_{l}} \longrightarrow & \left(P_{i_{1}}-c_{i_{1}}(h)\right) \cdots \\
& \left(P_{i_{k}}-c_{i_{k}}(h)\right) \otimes\left(X_{j_{1}} \cdots X_{j_{l}}\right),
\end{aligned}
$$

with $x_{j_{1}} \cdots x_{j_{l}} \in \mathcal{B} . \psi$ defines an algebraic star product on $\mathbb{C}\left[\mathfrak{g}^{*}\right][[h]]$, that we will denote by $\star_{P}$. Since $\psi$ descends to the quotient, it also defines an algebraic star product on $\mathbb{C}[\Theta][[h]]$ and we will denote it by $\star_{P \Theta}$. The case with $c_{i}(h)=c_{i}^{0}$ was considered first in Ref. [7], where it was shown that the star product is not differential.

\section{Comparison between $\star_{T \Theta}$ and $\star_{P \Theta}$}

In this section we want to compare the differential star product $\star_{T \Theta}$ and the algebraic star product $\star_{P \Theta}$ defined on a fixed regular coadjoint orbit $\Theta$. We want to show that there is an injective algebra homomorphism

$$
\tilde{H}:\left(\mathbb{C}[\Theta][[h]], \star_{P \Theta}\right) \longrightarrow\left(C^{\infty}(\Theta)[[h]]_{\mathbb{C}}, \star_{T \Theta}\right) .
$$

We will first show that there exists an injective algebra homomorphism

$$
H:\left(\mathbb{C}\left[\mathfrak{g}^{*}\right][[h]], \star_{P}\right) \longrightarrow\left(C^{\infty}\left(\mathcal{N}_{\Theta}\right)[[h]]_{\mathbb{C}}, \star_{T}\right),
$$

and then we will show that it descends appropriately to the quotients as an injective homomorphism.

In order to compare the tangential star products $\star_{P}$ on $\mathfrak{g}^{*}$ (algebraic, not differential) and $\star_{T}$ on $\mathcal{N}_{\Theta}$ (not algebraic, differential) we will use the non tangential star product $\star_{S}$ on $\mathfrak{g}^{*}$ (algebraic and differential).

The algebraic star products $\star_{P}$ and $\star_{S}$ on $\mathfrak{g}^{*}$ are equivalent, since they define algebra structures that are isomorphic to $U_{h}$. The equivalence is realized by the $\mathbb{C}[[h]]$-module isomorphism:

$$
\begin{gathered}
\eta:\left(\mathbb{C}\left[\mathfrak{g}^{*}\right][[h]], \star_{P}\right) \rightarrow\left(\mathbb{C}\left[\mathfrak{g}^{*}\right][[h]], \star_{S}\right) \\
\eta=\operatorname{Sym}^{-1} \circ \psi, \quad \eta\left(f \star_{P} g\right)=\eta(f) \star_{S} \eta(g) .
\end{gathered}
$$


By the very definition (四)

$$
f \star_{P} p_{i}=f \cdot p_{i},
$$

so, since the $p_{i}$ 's are central the ideal $\mathcal{I}_{0}=\left(p_{i}-c_{i}^{0}\right)$ in $\mathbb{C}\left[\mathfrak{g}^{*}\right][[h]]$ with respect to the commutative product is equal to the ideal with respect to the product $\star_{P}, \mathcal{I}_{0}^{\star_{P}}=\left(p_{i}-c_{i}^{0}\right)_{\star_{P}}$.

The generators of the ideal are mapped as

$$
\eta\left(p_{i}-c_{i}^{0}\right)=\left(\operatorname{Sym}^{-1} \circ \psi\right)\left(p_{i}-c_{i}^{0}\right)=\operatorname{Sym}^{-1}\left(P_{i}-c_{i}(h)\right)=p_{i}-c_{i}(h),
$$

so the ideal $\mathcal{I}_{0}^{\star P}$ is mapped isomorphically by $\eta$ onto the ideal with respect to the product $\star_{S}, \mathcal{I}_{c(h)}^{\star_{S}}=\left(p_{i}-c_{i}(h)\right)_{\star_{S}}$. We note that in the case of $\star_{S}, \mathcal{I}_{c(h)}$, the ideal generated by $p_{i}-c_{i}(h)$ with respect to the commutative product does not coincide with $\mathcal{I}_{c(h)}^{\star s}$. Notice also that one can choose the $c_{i}(h)$ 's arbitrarily, provided that $c_{i}(0)=c_{i}^{0}$.

Since $\star_{S}$ is differential, it is well defined on the whole $C^{\infty}\left(\mathfrak{g}^{*}\right)[[h]]_{\mathbb{C}}$. The commutative ideal generated by $p_{i}-c_{i}^{0}$ on $C^{\infty}\left(\mathfrak{g}^{*}\right)[[h]]_{\mathbb{C}}$ will be denoted by $\hat{\mathcal{I}}_{0}$. More generally, we can define $\mathcal{I}_{c(h)}=\left(p_{i}-c_{i}(h)\right) \subset \mathbb{C}\left[\mathfrak{g}^{*}\right][[h]], \hat{\mathcal{I}}_{c(h)}=$ $\left(p_{i}-c_{i}(h)\right) \subset C^{\infty}\left(\mathfrak{g}^{*}\right)[[h]]_{\mathbb{C}}$. We have that $\mathcal{I}_{0} \subset \hat{\mathcal{I}}_{0}$ and $\mathcal{I}_{c(h)} \subset \hat{\mathcal{I}}_{c(h)}$.

Let us consider the restriction map:

$$
r: C^{\infty}\left(\mathfrak{g}^{*}\right)[[h]]_{\mathbb{C}} \longrightarrow C^{\infty}\left(\mathcal{N}_{\Theta}\right)[[h]]_{\mathbb{C}} .
$$

Since the commutative product and $\star_{S}$ are both local, the restriction $r$ is an algebra homomorphism, between $C^{\infty}\left(\mathfrak{g}^{*}\right)[[h]]_{\mathbb{C}}$ and $C^{\infty}\left(\mathcal{N}_{\Theta}\right)[[h]]_{\mathbb{C}}$ as commutative algebras, and also between $\left(C^{\infty}\left(\mathfrak{g}^{*}\right)[[h]]_{\mathbb{C}}, \star_{S}\right)$ and $\left(C^{\infty}\left(\mathcal{N}_{\Theta}\right)[[h]]_{\mathbb{C}}, \star_{S \mathcal{N}_{\Theta}}\right)$.

We consider the restriction of polynomials $r\left(\mathbb{C}\left[\mathfrak{g}^{*}\right][[h]]\right)$. Since a polynomial is determined by its values on any open set, we can identify via $r$ $\left(\mathbb{C}\left[\mathfrak{g}^{*}\right][[h]], \star_{S}\right)$ with a subalgebra of $\left(C^{\infty}\left(\mathcal{N}_{\Theta}\right)[[h]]_{\mathbb{C}}, \star_{S \mathcal{N}_{\Theta}}\right)$.

On $C^{\infty}\left(\mathcal{N}_{\Theta}\right)[[h]]_{\mathbb{C}}$ there is an equivalence among $\star_{S \mathcal{N}_{\Theta}}$ and $\star_{T}$ (proposition 3.1). We denote it by

$$
\begin{aligned}
& \rho:\left(C^{\infty}\left(\mathcal{N}_{\Theta}\right)[[h]]_{\mathbb{C}}, \star_{S \mathcal{N}_{\Theta}}\right) \longrightarrow\left(C^{\infty}\left(\mathcal{N}_{\Theta}\right)[[h]]_{\mathbb{C}}, \star_{T}\right), \\
& \rho\left(f \star_{S} g\right)=\rho(f) \star_{T} \rho(g), \quad \rho=\operatorname{Id}+\sum_{n=1}^{\infty} h^{n} \rho_{n},
\end{aligned}
$$

where $\rho_{n}$ are bidifferential operators. We have given the injective homomorphism (5) by $H=\rho \circ r \circ \eta$.

We want now to show that $H\left(\mathcal{I}_{0}\right)=\hat{\mathcal{J}}_{0}$, where $\hat{\mathcal{J}}_{0}$ is the ideal with respect to $\star_{T}$ in $C^{\infty}\left(\mathcal{N}_{\Theta}\right)[[h]]_{\mathbb{C}}$ generated by $p_{i}-c_{i}^{0}$. 
We want to find out how the generators $p_{i}-c_{i}(h)$ are mapped under $\rho$. The scalars are mapped into scalars, since the bidifferential operators involved in the star products $\star_{T}$ and $\star_{S \mathcal{N}_{\Theta}}$ are null on the constants, and so are the operators $\rho_{n}$. We need to know $\rho\left(p_{i}\right)$.

\section{Remark 4.1}

Since $\rho$ is an isomorphism of algebras and $p_{i}$ belongs to the center of $\left(C^{\infty}\left(\mathcal{N}_{\Theta}\right)[[h]], \star_{S}\right), \rho\left(p_{i}\right)$ must also be in the center of $\left(C^{\infty}\left(\mathcal{N}_{\Theta}\right)[[h]], \star_{T}\right)$. A function $f$ in the center of $\left(C^{\infty}\left(\mathcal{N}_{\Theta}\right)[[h]], \star_{T}\right)$ is a function depending only on the global coordinates $f\left(p_{1}, \ldots p_{m}\right)$, since the condition

$$
f \star_{T} g-g \star_{T} f=0 \quad \forall g \in C^{\infty}\left(\mathcal{N}_{\Theta}\right)
$$

implies for the Poisson bracket

$$
\{f, g\}=0 \quad \forall g \in C^{\infty}\left(\mathcal{N}_{\Theta}\right),
$$

so $\{f$,$\} is a null Hamiltonian vector field and in particular does not have$ components tangent to the symplectic leaves.

\section{Remark 4.2}

The algebra homomorphism condition determines the form of $\rho$ on the center in terms of $\rho\left(p_{i}\right)=p_{i}+a_{i}(p, h)$, where $a_{i}(p, h)=h z_{i}(p, h)$. In fact, on the center we have

$$
\rho=\sum_{j_{1} \ldots j_{m}} a_{1 \ldots m}^{j_{1} \ldots j_{m}}(p, h) \frac{\partial}{\partial p_{1}^{j_{1}}} \cdots \frac{\partial}{\partial p_{m}^{j_{m}}} .
$$

$a_{1 \ldots m}^{0 \ldots 0}=1$ and the rest of coefficients are multiples of $\mathrm{h}$. In particular, the images of $p_{i}$ are $\rho\left(p_{i}\right)=p_{i}+a_{1 \ldots i \ldots m}^{0 \ldots \ldots .}(p, h)=p_{i}+a_{i}(p, h)$. Using the fact that $\star_{T}$ is tangential we have

$$
f \star_{T} p_{i}=f \cdot p_{i} \quad \forall f \in C^{\infty}\left(\mathcal{N}_{\Theta}\right),
$$

the homomorphism condition reads

$$
\rho\left(p_{1}^{i_{1}} \cdots p_{m}^{i_{m}}\right)=\left(p_{1}+a_{1}\right)^{i_{1}} \cdots\left(p_{m}+a_{m}\right)^{i_{m}} .
$$

The solution of this equation is

$$
a_{1 \ldots m}^{j_{1} \ldots j_{m}}=\frac{1}{j_{1} ! \cdots j_{m} !} a_{1}^{j_{1}} \cdots a_{m}^{j_{1}} .
$$


In particular, $\rho$ is trivial on the center if and only if $a_{1}=\cdots=a_{m}=0$.

By remark 6.1 we have that

$H\left(p_{i}-c_{i}^{0}\right)=\rho\left(p_{i}-c_{i}(h)\right)=p_{i}-a_{i}(p, h)-c_{i}(h)=p_{i}-c_{i}^{0}+h\left(z_{i}(p, h)-\Delta_{i}(h)\right)$,

where we have denoted $a_{i}(p, h)=h z_{i}(p, h)$ and $c_{i}(h)=c_{i}^{0}+h \Delta_{i}(h)$. Since $\Delta_{i}(h)$ is arbitrary, we can choose it as

$$
\Delta_{i}(h)=z_{i}\left(c_{i}^{0}, h\right) .
$$

It is not hard to see that

$$
z_{i}(p, h)-\Delta_{i}(h)=\sum_{j=1}^{m} b_{i j}\left(p_{j}-c_{j}^{0}\right) \in r\left(\mathcal{I}_{0}\right),
$$

and we have

$$
p_{i}-c_{i}^{0}+h\left(z_{i}(p, h)-\Delta_{i}(h)\right)=\sum_{j=1}^{m}\left(\delta_{i j}+h b_{i j}\right)\left(p_{i}-c_{i}^{0}\right) .
$$

The matrix $\left(\delta_{i j}+h b_{i j}\right)$ is invertible, so the ideal generated by $H\left(p_{i}-c_{i}^{0}\right)$ in $H\left(\mathbb{C}\left[\mathfrak{g}^{*}\right][[h]]\right)$ coincides with the ideal generated by $\left(p_{i}-c_{i}^{0}\right)$ in $H\left(\mathbb{C}\left[\mathfrak{g}^{*}\right][[h]]\right)$. (For $\star_{T}$, the star ideal coincides with the commutative ideal).

In order to state the main result we need a lemma.

Lemma 4.1 Let $\mathcal{J}_{0}$ be the ideal in $\left(H\left(\mathbb{C}\left[\mathfrak{g}^{*}\right][[h]]\right), \star_{T}\right)$ generated by $\left(p_{i}-c_{i}^{0}\right)$ and let $\hat{\mathcal{J}}_{0}$ be the ideal in $\left(C^{\infty}\left(\mathcal{N}_{\Theta}\right)[[h]], \star_{T}\right)$ generated by the same generators. Then

$$
\hat{\mathcal{J}}_{0} \cap H\left(\mathbb{C}\left[\mathfrak{g}^{*}\right][[h]]\right)=\mathcal{J}_{0}
$$

Proof. Since the product $\star_{T}$ is tangential to the orbits the star ideals $\mathcal{J}_{0}$ and $\hat{\mathcal{J}}_{0}$ coincide with the ideals with respect to the commutative product, so we will limit ourselves to those.

One inclusion is obvious. For the other, let $b=\sum_{r=0}^{\infty} b_{r} h^{r} \in H\left(\mathbb{C}\left[\mathfrak{g}^{*}\right][[h]]\right)$. Assume that

$$
H(b)=\sum_{i=1}^{m} f^{i} H\left(p_{i}-c_{i}^{0}\right)
$$


where $f^{i}=\sum_{r=0}^{\infty} f_{r}^{i} h^{r} \in C^{\infty}\left(\mathcal{N}_{\Theta}\right)[[h]]_{\mathbb{C}}$ are not unique. We need to prove that $f^{i}$ can be chosen in $H\left(\mathbb{C}\left[\mathfrak{g}^{*}\right][[h]]\right)$. We will show that there exist $q^{i}=$ $\sum_{r=0}^{\infty} q_{r}^{i} h^{r} \in \mathbb{C}\left[\mathfrak{g}^{*}\right][[h]]$ such that $b_{r}=\sum_{i=1}^{m} q_{r}^{i}\left(p_{i}-c_{i}^{0}\right)$. This clearly will be enough.

By induction on $r$. For $r=0$, we look at the order 0 in $h$ of the equation (8) (we recall that $H=\operatorname{Id} \bmod (h)$ )

$$
b_{0}=\sum_{i} f_{0}^{i}\left(p_{i}-c_{i}^{0}\right) \in \mathbb{C}\left[\mathfrak{g}^{*}\right] \subset C^{\infty}\left(\mathcal{N}_{\Theta}\right)_{\mathbb{C}}
$$

It is not hard to see that $f_{0}^{i}$ can be chosen in $\mathbb{C}\left[\mathfrak{g}^{*}\right]$, so we set $q_{0}^{i}=f_{0}^{i}$.

We go to the general case. By the induction hypothesis, we assume that we have found $q_{0}^{i}, \ldots q_{r}^{i}$, with

$$
b_{0}+b_{1} h+\cdots+b_{r} h^{r}=\sum_{i=1}^{m}\left(q_{0}^{i}+q_{1}^{i} h+\cdots+q_{r}^{i} h^{r}\right)\left(p_{i}-c_{i}^{0}\right) .
$$

Then,

$$
H(b)-H\left(b_{0}+b_{1} h+\cdots+b_{r} h^{r}\right)=\sum_{i=1}^{m}\left(f^{i}-H\left(q_{0}^{i}+\cdots+q_{r}^{i} h^{r}\right) H\left(p_{i}-c_{i}^{0}\right),\right.
$$

So

$$
h^{r+1} H\left(b_{r+1}+b_{r+2} h+\cdots\right)=h^{r+1} \sum_{i=1}^{m}\left(f_{r+1}^{i}-\sum_{s+t=r+1} H_{s}\left(q_{t}^{i}\right)\right) H\left(p_{i}-c_{i}^{0}\right) \bmod \left(h^{r+2}\right) .
$$

Since the ring $C^{\infty}\left(\mathcal{N}_{\Theta}\right)[[h]]_{\mathbb{C}}$ is torsion free we have:

$$
H\left(b_{r+1}+b_{r+2} h+\cdots\right)=\sum_{i=1}^{m}\left(f_{r+1}^{i}-\sum_{s+t=r+1} H_{s}\left(q_{t}^{i}\right)\right) H\left(p_{i}-c_{i}^{0}\right) \bmod (h) .
$$

Now if we look at the order 0 in $h$

$$
b_{r+1}=\sum_{i=1}^{m}\left(f_{r+1}^{i}-\sum_{s+t=r+1} H_{s}\left(q_{t}^{i}\right)\right)\left(p_{i}-c_{i}^{0}\right),
$$

as in the $r=0$ case, if we set

$$
q_{r+1}^{i}=f_{r+1}^{i}-\sum_{s+t=r+1} H_{s}\left(q_{t}^{i}\right)
$$

it is not hard to see that it can be chosen as a polynomial, which gives us the result. 
Proposition 4.1 Let $\Theta$ be a regular coadjoint orbit of a compact Lie group defined by the constrains

$$
p_{i}-c_{i}^{0}, \quad i=1, \ldots m
$$

There is an injective homomorphism between the algebraic deformation of $\mathbb{C}[\Theta]$ defined by $U_{h} / \mathcal{I}_{h}$ with $\mathcal{I}_{h}$ generated by

$$
P_{i}-c_{i}^{0}+h \Delta_{i}(h)
$$

and the differential deformation of $C^{\infty}(\Theta)_{\mathbb{C}}\left(C^{\infty}(\Theta)[[h]]_{\mathbb{C}}, \star_{T \Theta}\right)$, which is obtained via Kontsevich formula (see $§ 5$ for more details), provided the constants $\Delta_{i}(h)$ are chosen as in (W).

Proof. $H$ is an algebra isomorphism onto its image. We have the commutative diagram

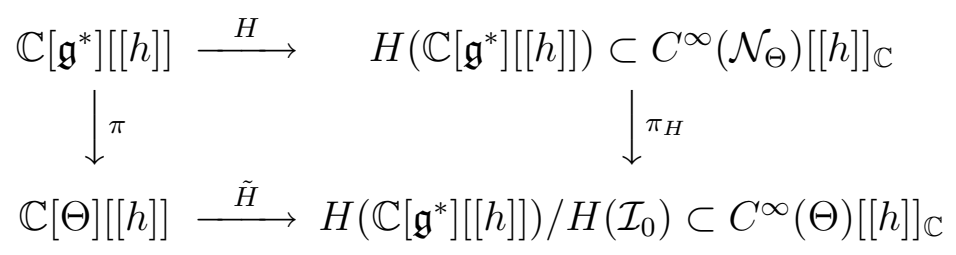

The last inclusion follows from 4.1.

\section{Remark 4.3}

We want to note that the ideal $\mathcal{I}_{h}$ used in the previous proposition is not in general the ideal used in geometric quantization. In fact for $\mathrm{SU}(2)$ it was shown in Ref. [8] that the latter is generated by

$$
P-l(l+\hbar), \quad \hbar=\frac{h}{2 \pi}
$$

( $P$ is the Casimir of $\mathfrak{s u}(2)$ ). But by the remark 4.2, (6) the ideal has, either $c_{i}(h)=c_{i}^{0}$ or $\Delta^{i}(h)$ is an infinite series in $h$ (an exponential). Then we have a contradiction. 


\section{Appendix A}

In this appendix we want to give some standard definitions on deformations and star products that have been use throughout the text.

Definition A1 Let $(\mathcal{A},\{\}$,$) be a Poisson algebra over \mathbb{R}$. We say that the associative algebra $\mathcal{A}_{[h]}$ over $\mathbb{R}[[h]]$ is a formal deformation of $\mathcal{A}$ if

1. There exists an isomorphism of $\mathbb{R}[[h]]$-modules $\psi: \mathcal{A}[[h]] \longrightarrow \mathcal{A}_{[h]}$;

2. $\psi\left(f_{1} f_{2}\right)=\psi\left(f_{1}\right) \psi\left(f_{2}\right) \bmod (\mathrm{h}), \quad \forall f_{1}, f_{2} \in A[[h]]$;

3. $\psi\left(f_{1}\right) \psi\left(f_{2}\right)-\psi\left(f_{2}\right) \psi\left(f_{1}\right)=h \psi\left(\left\{f_{1}^{0}, f_{2}^{0}\right\}\right) \bmod \left(h^{2}\right), \quad \forall f_{1}, f_{2} \in \mathcal{A}[[h]]$, $f_{i} \equiv f_{i}^{0} \bmod (h), i=1,2$.

If $\mathcal{A}_{\mathbb{C}}$ is the complexification of a real Poisson algebra $\mathcal{A}$ we can give the definition of formal deformation of $\mathcal{A}_{\mathbb{C}}$ by replacing $\mathbb{R}$ with $\mathbb{C}$ in the above definition.

The associative product in $\mathcal{A}[[h]]$ defined by:

$$
f \star g=\psi^{-1}(\psi(f) \cdot \psi(g)), \quad f, g \in \mathcal{A}[[h]]
$$

is called the star product on $\mathcal{A}[[h]]$ induced by $\psi$.

A star product on $\mathcal{A}[[h]]$ can be also defined as an associative $\mathbb{R}[[h]]$-linear product given by the formula:

$$
f \star g=f g+B_{1}(f, g) h+B_{2}(f, g) h^{2}+\cdots \in \mathcal{A}[[h]], \quad f, g \in \mathcal{A}
$$

where the $B_{i}$ 's are bilinear operators. By associativity of $\star$ one has that $\{f, g\}=B_{1}(f, g)-B_{1}(g, f)$. So this definition is a special case of the previous one where $\mathcal{A}_{h}=\mathcal{A}[[h]]$ and $\star$ is induced by $\psi=\mathrm{Id}$.

Two star products on $\mathcal{A}[[h]], \star$ and $\star^{\prime}$ are said to be equivalent (or gauge equivalent) if there exists $T=\sum_{n \geq 0} h^{n} T_{n}$, with $T_{n}$ linear operators on $\mathcal{A}[[h]]$, $T_{0}=$ Id such that

$$
f \star g=T^{-1}\left(T f \star^{\prime} T g\right) .
$$

If $\mathcal{A} \subset C^{\infty}(M)$ and the operators $B_{i}$ 's are bidifferential operators we say that the star product is differential. If in addition $\mathcal{A}=C^{\infty}(M)$ and $M$ is a real Poisson manifold, we will say that $\star$ is a differential star product on $M$.

In [1] Kontsevich classifies differential star products on a manifold $M$ up to gauge equivalence.

Theorem A1 (Kontsevich, [1]) The set of gauge equivalence classes of differential star products on a smooth manifold $M$ can be naturally identified 
with the set of equivalence classes of Poisson structures depending formally on $h$,

$$
\alpha=h \alpha_{1}+h^{2} \alpha_{2}+\cdots
$$

modulo the action of the group of formal paths in the diffeomorphism group of $M$, starting at the identity isomorphism.

In particular, for a given Poisson structure $\alpha_{1}$, we have the equivalence class of differential star products associated to $h \alpha_{1}$. We will say that this is the equivalence class of star products canonically associated to the Poisson structure $\alpha_{1}$.

Also, an explicit universal formula to compute the bidifferential operators of the star product associated to any formal Poisson structure was given in Ref. [1] in the case of an arbitrary Poisson structure on flat space $\mathbb{R}^{n}$. The formula depends on the coordinates chosen, but it was also proven in Ref. [1] that the star products constructed with different choices of coordinates are gauge equivalent.

Let $\mathcal{A}_{\mathbb{C}}=\mathbb{C}\left[M_{\mathbb{C}}\right]$ be the coordinate ring of the complex algebraic affine variety $M_{\mathbb{C}}$ defined over $\mathbb{R}$ whose real points are a real algebraic Poisson variety $M$. If the $B_{i}$ 's are bilinear algebraic operators we will say that $\star$ is an algebraic star product on $M$.

An example of great interest for us, of such $M$ is given by the dual $\mathfrak{g}^{*}$ of the Lie algebra of a compact semisimple Lie group (see section 2).

The classification of algebraic star products is still an open problem [11.

Definition A2 Let $N$ be a submanifold of the Poisson manifold $M$ and let $\star_{M}$ be a star product on $M$. We say that $\star_{M}$ is tangential to $N$ if for $f, g \in C^{\infty}(N)$ :

$$
f \star_{N} g=\left.{ }_{\text {def }}\left(F \star_{M} G\right)\right|_{N}, \text { with } f=\left.F\right|_{N}, \quad g=\left.G\right|_{N}
$$

is a well defined star product on $N$, that is, if

$$
\left.\left(F-F^{\prime}\right)\right|_{N}=\left.\left(G-G^{\prime}\right)\right|_{N}=0, \text { then }\left.\quad F \star_{M} G\right|_{N}=\left.F^{\prime} \star_{M} G^{\prime}\right|_{N},
$$

for $F, F^{\prime}, G, G^{\prime} \in C^{\infty}(M)$.

The same definition works for algebraic Poisson varieties, replacing the algebra of $C^{\infty}$ functions with the algebra of polynomials on the varieties. 
Given a Poisson manifold $M$, one can ask if there exists a differential star product on $M$, that is tangential to all the leaves of the symplectic foliation. For regular manifolds a positive answer was found in Ref. [6]. For $M=\mathfrak{g}^{*}$ foliated in coadjoint orbits, it was found in Ref. 13 that there is an obstruction to the existence. In particular, for a semisimple Lie algebra $\mathfrak{g}^{*}$ it is not possible to find a differential star product on $\mathfrak{g}^{*}$ which is tangential to all coadjoint orbits.

\section{Appendix B}

In this Appendix we want to give an explicit formula on how to construct a global star product starting from star products defined on open sets of a manifold and satisfying certain conditions (see below). We will refer to this procedure as gluing of star products, and it will be used in section 3 .

Let $\star$ be a differential star product on a manifold $M$. Since the operators $B_{i}$ that define $\star$ are local, there are well defined star products $\star_{U}$ on every open set $U$ of $M$. We have a sheaf of algebras $\mathcal{S}$ :

$$
\mathcal{S}(U)=\left(\mathbb{C}^{\infty}(U)[[h]], \star_{U}\right) .
$$

which we will call sheaf of star products.

Let $M$ be a Poisson manifold and fix an open cover $\mathcal{U}=\left\{U_{r}\right\}_{r \in J}$ where $J$ is some set of indices. Assume that in each $U_{r}$ there is a differential star product

$$
\star_{r}: C^{\infty}\left(U_{r}\right)[[h]] \otimes C^{\infty}\left(U_{r}\right)[[h]] \longrightarrow C^{\infty}\left(U_{r}\right)[[h]] .
$$

This defines a collection of sheaves of star products

$$
\mathcal{F}_{r}\left(V_{r}\right)=\left(C^{\infty}\left(V_{r}\right)[[h]], \star_{r}\right), \quad V_{r} \subset U_{r} .
$$

It is a general fact in theory of sheaves that if there are isomorphisms of sheaves in the intersections

$$
\begin{aligned}
& T_{s r}: \mathcal{F}_{r}\left(U_{r s}\right) \longrightarrow \mathcal{F}_{s}\left(U_{s r}\right), \quad U_{r_{1} \ldots r_{k}}=U_{r_{1}} \cap \cdots \cap U_{r_{k}} \\
& T_{s r}(f) \star_{s} T_{s r}(g)=T_{s r}\left(f \star_{r} g\right)
\end{aligned}
$$

such that the following conditions are satisfied

$$
\begin{aligned}
& \text { 1. } T_{r s}=T_{s r}^{-1} \quad \text { on } U_{s r} \text {, } \\
& \text { 2. } T_{t s} \circ T_{s r}=T_{t r}, \quad \text { on } U_{r s t} \text {, }
\end{aligned}
$$


then there exists a global sheaf $\mathcal{F}$ on $M$ isomorphic to the local sheaves $\mathcal{F}_{r}$ on each $U_{r}$.

If the sheaves of star products (12) satisfy the conditions (14) with

$$
T_{s r}=\operatorname{Id} \bmod (h),
$$

then we have a global sheaf of star products on $M$. The algebra of the global sections is $C^{\infty}(M)[[h]]$ together with a star product that we will call the gluing of local star products. We want to write an explicit formula for the star product of global sections.

We denote $U^{r_{1} \ldots r_{k}}=U_{r_{1}} \cup \cdots \cup U_{r_{k}}$. Let us first consider the gluing on two open sets, say $U_{1}$ and $U_{2}$, with non trivial intersection. Let $\phi_{1}: U_{1} \rightarrow \mathbb{R}$, $\phi_{2}: U_{2} \rightarrow \mathbb{R}$ be a partition of unity of $U^{12}$,

$$
\phi_{1}(x)+\phi_{2}(x)=1 \quad \forall x \in U^{12} ; \quad \operatorname{supp}\left(\phi_{r}\right) \subset U_{r} .
$$

Let $f_{r} \in C^{\infty}\left(U_{r}\right)[[h]]$ such that $f_{s}=T_{s r} f_{r}$ in $U_{r s}$. One can define an element $f \in C^{\infty}\left(U^{r s}\right)[[h]]$ by $f=\phi_{1} f_{1}+\phi_{2} f_{2}$. On the intersection $U_{12}$ one has

$$
f=\phi_{1} f_{1}+\phi_{2} T_{21} f_{1}=\left(\phi_{1} \mathrm{Id}+\phi_{2} T_{21}\right) f_{1}=A_{21} f_{1} .
$$

Notice that the operator $A_{21}=\operatorname{Id}+\mathcal{O}(h)$ is invertible. On $U^{12}$ we can define the star product

$$
f \star g= \begin{cases}\left(f_{1} \star_{1} g_{1}\right)(x) & \text { if } x \in U_{1}-U_{12} \\ A_{21}\left(A_{21}^{-1}(f) \star_{1} A_{21}^{-1}(g)\right)(x) & \text { if } x \in U_{12} \\ \left(f_{2} \star_{2} g_{2}\right)(x) & \text { if } x \in U_{2}-U_{12}\end{cases}
$$

It is easy to check that the star product is smooth.

One can do the gluing interchanging $U_{1}$ and $U_{2}$. One has that on $U_{12}$

$$
f=\phi_{1} f_{1}+\phi_{2} f_{2}=\left(\phi_{1} T_{12}+\phi_{2} \mathrm{Id}\right) f_{j}=A_{12} f_{2} .
$$

$A_{12}$ is also invertible and

$$
A_{21}=A_{12} T_{21}
$$

provided $T_{12}=T_{21}^{-1}$. One can construct a star product on $U^{12}$ using the same procedure than in (15). It is easy to check that both star products are identical. 
The procedure in (15) can be generalized to an arbitrary number of open sets. Let $\phi_{i}: U_{i} \rightarrow \mathbb{R}$ a partition of unity of $M$ subordinate to the covering $\mathcal{U}$. We define $f \in C^{\infty}(M)$

$$
f=\sum_{r \in J} \phi_{r} f_{r}, \quad \text { where } f_{r}=T_{r s} f_{s} .
$$

On $U_{r} f$ becomes

$$
f=\left(\phi_{r} \mathrm{Id}+\sum_{s} \phi_{s} T_{s r}\right) f_{r}=A_{r} f_{r} .
$$

The star product on $U_{r}$ is defined as

$$
f \star g=A_{r}\left(A_{r}^{-1}(f) \star_{r} A_{r}^{-1}(g)\right) .
$$

Using conditions (14) one has

$$
A_{r} T_{r t}=A_{t}
$$

Then, the star products (16) on each $U_{r}$ coincide in the intersections, so they define a unique star product on $M$. The restriction of this star product to $U_{r}$ is equivalent to $\star_{r}$. Also, using different partitions of unity one obtains equivalent star products.

\section{Acknoledgements}

We wish to thank A. Levrero for helpful discussions.

\section{References}

[1] M. Kontsevich, Deformation Quantization of Poisson Manifolds. math.QA/9709040.

[2] H. Omori, Y. Maeda and A. Yoshioka, Weyl Manifolds and Deformation Quantization. Adv. in Math. 85 224-255 (1991).

[3] M. De Wilde, P. B. A. Lecomte, Existence of Star Products and of Formal Deformations of the Poisson Lie Algebra of Arbitrary Symplectic Manifolds. Lett. Math. Phys. 7 487-49 (1983). 
[4] B. Fedosov, A Simple Geometric Construction of Deformation Quantization. J. Diff. Geom. 40 Vol. 2 213-238 (1994).

[5] P. Deligne, Déformations de l'Algebre des Fonctions d'une Variété Symplectique: Comparaison entre Fedosov et De Wilde, Lecomte Selecta Math. New series 1 No.4 667-697 (1995).

[6] M. Masmoudi, Tangential deformation of a Poisson bracket and tangential star-products on a regular Poisson manifold. J. Geom. Phys. 9 155-171 (1992).

[7] M. Cahen, S. Gutt, Produits * sur les Orbites des Groupes Semi-Simples de Rang 1, C.R. Acad. Sc. Paris 296 (1983), série I, 821-823; An Algebraic Construction of * Product on the Regular Orbits of Semisimple Lie Groups. In "Gravitation and Cosmology". Monographs and Textbooks in Physical Sciences. A volume in honor of Ivor Robinson, Bibliopolis. Eds W. Rundler and A. Trautman, (1987); Non Localité d'une Déformation Symplectique sur la Sphère $S^{2}$. Bull. Soc. Math. Belg. 36 B 207-221 (1987).

[8] R. Fioresi and M. A. Lledó, On the deformation Quantization of Coadjoint Orbits of Semisimple Lie Groups. To appear in the Pacific J. of Math. math.QA/9906104.

[9] R. Fioresi, A. Levrero and M. A. Lledó, Algebraic and Differential Star Products on Regular Orbits of Compact Lie Groups. To appear in the Pacific J. of Math. math.QA/0011172.

[10] M. A . Lledó, Deformation Quantization of Non Regular Orbits of Compact Lie Groups. math.QA/0105191.

[11] M. Kontsevich, Deformation Quantization of algebraic varieties. math.AG/0106006.

[12] B. Kostant, Lie Group Representations on Polynomial Rings. Am. J. Math. 85327 (1978).

[13] M. Cahen, S. Gutt, J. Rawnsley, On Tangential Star Products for the Coadjoint Poisson Structure. Comm. Math. Phys, 180 99-108 (1996). 